\title{
High Fidelity Modeling of 30m Near-Field Cassegrain Antenna in X-band
}

\author{
A. Di Maria ${ }^{1}$, L. Pandolfo ${ }^{2}$, P. De Vita ${ }^{2}$, G. Guida ${ }^{2}$, M. Bercigli ${ }^{2}$, M. Bandinelli ${ }^{2}$ \\ ${ }^{1}$ Microwaves and Radar Institute, German Aerospace Center (DLR), Oberpfaffenhofen, Germany, Alberto.DiMaria@dlr.de \\ ${ }^{2}$ Computational Electromagnetic Laboratory, IDS Ingegneria Dei Sistemi S.p.A., Pisa, Italy, m.bandinelli@idscorporation.com
}

\begin{abstract}
A $30 \mathrm{~m}$ Near Field Cassegrain antenna has been accurately simulated using a real $3 \mathrm{D}$ acquisition of the reflectors surfaces. Even though no kind of symmetry or approximation can be exploited, Antenna Design Framework (ADF) software is able to model this problem at $1.5 \mathrm{GHz}$ with a full-wave approach and even at $10 \mathrm{GHz}$ with a mixed-methods approach.
\end{abstract}

Index Terms - antenna, reflector, propagation, simulation, full-wave, 3D scan

\section{INTRODUCTION}

The Helios Command Station was built in 1974 in Weilheim by the German Aerospace Center (DLR) to serve as ground station for the Helios-A/B satellite missions. Together with the NASA deep space ground station, it maintained around the clock the link with the two space probes over a distance of up to 186 million miles ${ }^{1}$.

The Helios Command Station antenna has near-field Cassegrain configuration with a 30 meter diameter main reflector and with an elevation over azimuth mount. After the Helios mission came to an end, the antenna was used to support the scientific space missions Giotto, AMPTE and Equator-S as well as several scientific experiments. The antenna was originally designed to operate for the transmission of commands in the S-band $(2110-2120 \mathrm{MHz})$.

In 1974, right after the construction, DLR performed the mechanical alignment of the reflectors and the positioner accuracy verification, along with the electrical calibration and the pattern measurements. The sender antenna used for these electrical measurements was a $2 \mathrm{~m}$ parabolic reflector positioned on the Alps at a distance of $25 \mathrm{~km}$ and at a relative altitude of $760 \mathrm{~m}$.

There are some intrinsic limitations in measuring such a massive antenna with an Earth to Earth setup. The big reflector has to point the transmitting antenna with a low elevation angle and this makes it exposed to the interferences from other sources and to the multipath from the ground. In addition the structure deformation due to its own weight does not allow a proper elevation cut measure. To overcome these problems it is always preferable to point the reflector upwards and so, later on the same year, a second antenna radiation measurement was carried out using the senders of the ALSEP basis stations left on the Moon from the Apollo missions.

Despite this original intent no measurements were ever done in X-band. Moreover the original shape verification and reflector panels alignment were carried out using an optical theodolite. Even though they are used extensively in this kind of application, these geodesic techniques are unable to provide accuracy better than $1 \mathrm{~mm}$.

Recently a new approach has been tested: the reflectors surfaces have been acquired with a 3D laser scanner obtaining a dense and precise cloud of points.

The present paper describes how these data are successfully used to simulate the electromagnetic behavior of the antenna.

The purpose is to show that such simulation can be a valid alternative to the measurements. For this reason we retain every small detail of the scanned surfaces, although this wouldn't let us use any kind of symmetry to relax the problem complexity. This and the huge dimension of the problem - the main reflector diameter is $10^{3} \lambda$ at X-band - set unprecedented challenges to the simulation.

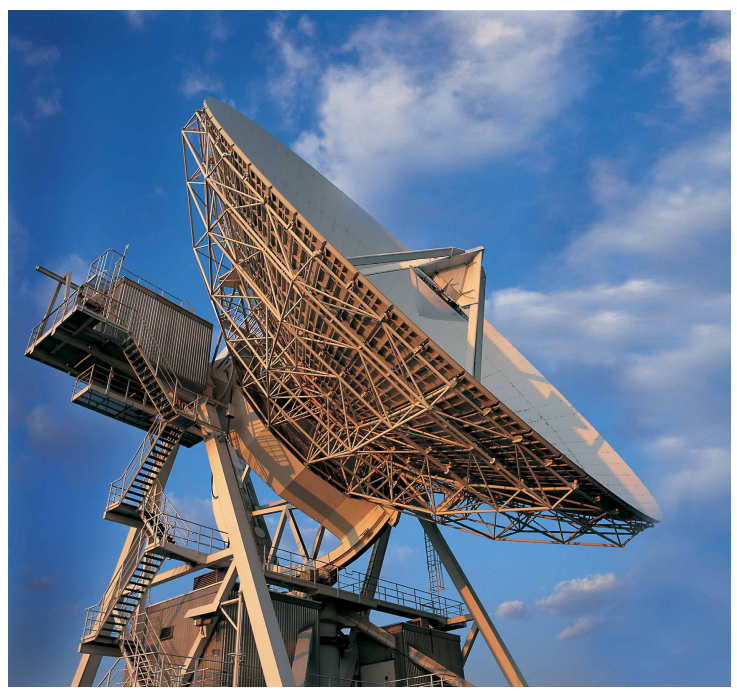

Fig. 1. The $30 \mathrm{~m}$ reflector antenna of the Helios Command Station in Weilheim.
1 HELIOS was the first US/German interplanetary mission. Launched in 1974 (HELIOS 1, 10 December 1974 - 15 March 1986) and 1976 (HELIOS 2, 15 January 1976 - 8 January 1981), the two German built (MBB) Helios probes approached the sun closer than the inner planet Mercury (0.3 AU) and closer than any spaceprobe ever. 


\section{ANTENNA GEOMETRY DESCRIPTION AND SURFACE ACQUISITION}

The Helios Command Station antenna has a Near Field Cassegrain setup. This configuration employs three reflectors and combines some of the useful properties of the standard Cassegrain with those of the horn reflector.

The standard Cassegrain has, in the usual arrangement, a point source feed located at the vertex of the main reflector, which illuminates with a spherical wave a hyperboloidal subreflector located near the focal plane.

The Near Field Cassegrain employs instead a plane wave feed, specifically a horn reflector, which is still located at the vertex of the main reflector, and has dimension comparable to the subreflector. The subreflector has a paraboloidal shape and it is illuminated by a uniform phase front [1]. An idealized sketch is presented in Fig. 2.

The main and the subreflector have diameters of $30 \mathrm{~m}$ and $4.1 \mathrm{~m}$ respectively. The circular horn reflector is about $9.4 \mathrm{~m}$ long and has an aperture diameter of $2.3 \mathrm{~m}$. The feed internal reflector is an elliptical section of a paraboloid and its main axis is $3.2 \mathrm{~m}$.

Two different 3D laser scanner models were used: the Leica HDS3000 and the Z+F IMAGER 5006h. The scanners were placed above the vertex of the main reflector with a sturdy structure that could firmly hold them above the horn reflector aperture.

The scanner automatically acquired millions of points for each of the three reflectors, resulting in a fairly dense cloud of points with a level of detail high enough to generate accurate simulation results. Unfortunately neither these points, nor the triangulations directly derivable from them, are suitable to be used directly for the simulation.

The faceted surface will constrain the simulation mesh generation process, resulting in a mesh that is bound and restricted to the original points and cannot be further refined.

The model used for the simulation is shown in Fig. 3.

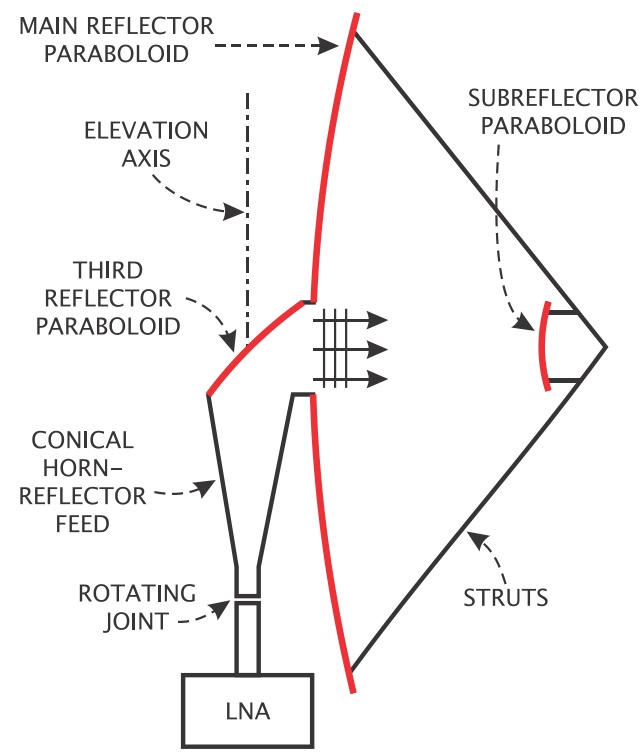

Fig. 2. Idealized sketch of the Near Field Cassegrain configuration.

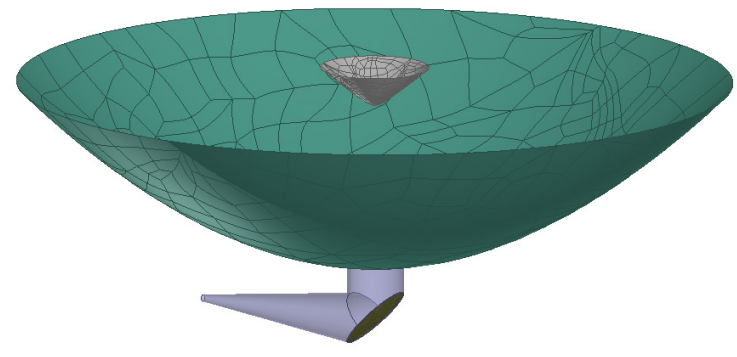

Fig. 3. The model of the antenna suited for the simulation.

The surfaces of all three reflectors are here defined by nurbs that fit the raw points obtained by the scans. The fitting process is done by a least square algorithm and the resulting surfaces have a maximum deviation of $0.1 \mathrm{~mm}$, whereas the mean deviation is less than $10^{-6} \mathrm{~mm}$.

\section{ADF MODELING PROCEDURES}

It is needless to say that the modeling of this specific reflector antenna requires a highly efficient approach to both working procedure and computational resources.

The analysis of the antenna in a frequency range extending from L-band to X-band entails the combined use of different modeling methods with the aim of optimizing the calculation performances without compromising the required results accuracy.

Antenna Design Framework (ADF) is an Electronic Design Automation (EDA) software tool dedicated to the antenna design and the antenna siting on complex platforms, which has been developed in collaboration with ESA/ESTEC Electromagnetics Division [2].

Several interoperable tools, based on various modeling and acceleration techniques, are combined in the framework. By cascading these different tools, it is possible to configure several computational processes, which greatly extend the single method capabilities.

The following sections describe the different computational approaches, which have been selected to analyze the antenna system shown in Fig. 3.

\section{A. Full-Wave "one-shot" Approach}

The full-wave "one-shot" approach is a procedure based on the Method of Moments (MoM). The whole antenna is considered as a linear multi-port system consisting of parts having very different resolution levels (e.g. feed details compared to those of the main reflector). Thanks to a MultiLevel Fast Multipole Approach (MLFMA) powered by means of a Multi-Resolution preconditioner [3][4], the entire system can be simulated at once (hence the name), taking consequently into account:

- the different propagation modes of the guiding structure;

- the coupling effects among all the parts of the system.

This approach is clearly the most accurate, but it has the drawback of not being able to scale up to X-band due to the huge computational complexity ( 250 million of unknowns). Given the high accuracy, the full wave "one-shot" results will be taken as the reference for the other methods. 


\section{B. Full-Wave "two-steps" Approach}

The full-wave "two-steps" approach is still a Method of Moment based procedure, but, unlike the full-wave "one-shot" case, the whole antenna system is here decoupled in two separated sub-systems by means of some intermediate model translations (i.e. using source equivalent models):

a. feed and waveguide system;

b. main and sub reflector system.

The decoupling operation is considered feasible because the interactions among the two sub-systems are expected to be as weak as to ignore the "feedback" effect of reflectors system on the feeding system.

It's worth noting that this approach retains the same computational complexity of the previous one and therefore it cannot be extended up to X-band. Despite this, the present approach is important for two main reasons:

- the first step of the procedure provides a full-wave equivalent model of the feeding system;

- the separation of the antenna in two decoupled parts can be validated through the present approach and then taken as basic assumption for the Mixed Methods procedure described later.

\section{Iterative Physical Optic}

Iterative Physical Optic (IPO) iteratively refines the Physical Optic (PO) currents to account for multiple reflections and diffractions within the high-frequency asymptotic assumptions of PO [5]. The basic approach is to start with the first-order PO currents, ignoring shadowing effects, and then iteratively integrate the currents by using a simple facet visibility check. The iteration is halted after a prescribed number of bounces are reached or the IPO currents converge.

The size of the problem solved in the present work $(\sim 38$ million of facets) requires parallel computing resources and a highly sophisticated software implementation. Moreover, acceleration techniques, like the Fast Far-Field Approximation (FaFFA), have been implemented in order to accelerate the matrix-vector products of the IPO formulation.

The convergence and the accuracy of the IPO method are demonstrated in the numerical results of Section IV by comparison with the reference full wave "one-shot" solution.

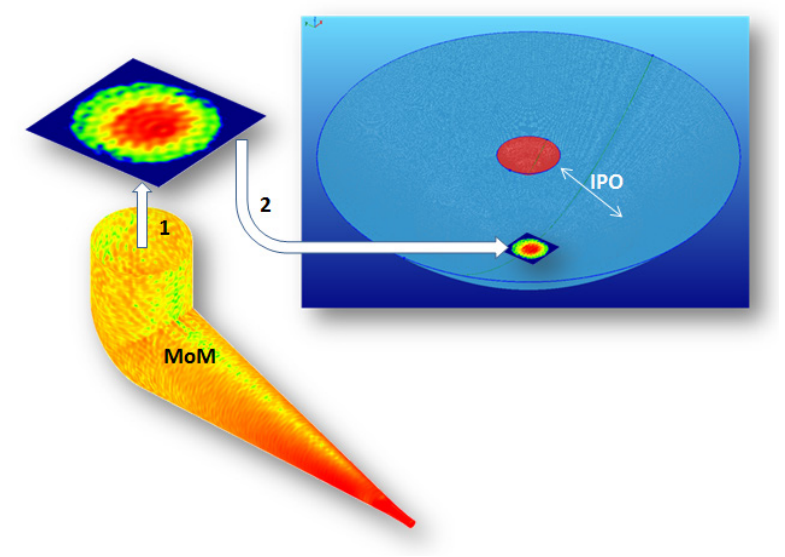

Fig. 4. Mixed-methods computational procedure.

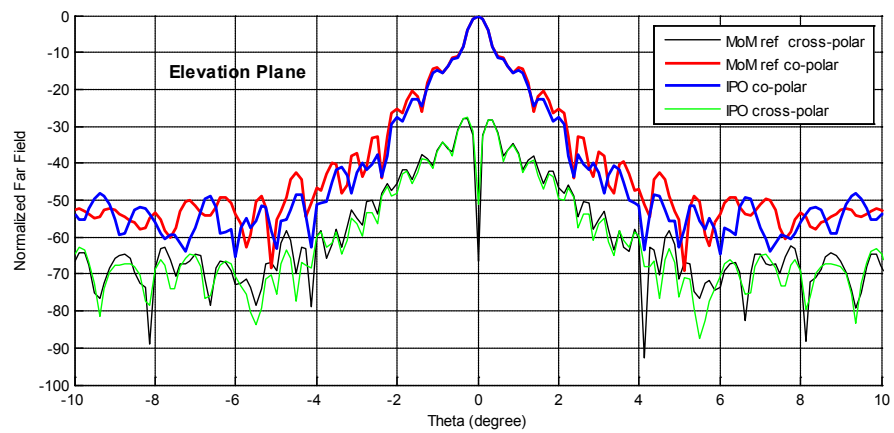

Fig. 5. Comparision between the Full-Wave "one-shot" results (the reference) and the Mixed-Methods results.

\section{Mixed-Methods Approach}

By cascading the full-wave analysis and the IPO method, it is possible to configure a computational process, referred to as Mixed-Methods approach, capable of solving the problem up to X-band. The whole antenna system is decoupled in the same two sub-systems described in method B, under the same assumption of weak interaction among the reflectors and the feed. MoM technique is applied to the feeding system, while the scattering from the double reflector is entrusted to the IPO tool (Fig. 4). It is the latter asymptotic high frequency method that extends our analysis capability up to X-band, still taking into account the full interaction between the two reflectors.

\section{METHOD VALIDATION}

The Mixed-Methods approach has been validated against the reference full wave "one-shot" solution in order to assess its accuracy. The pattern results obtained by the two different computational procedures at $1.5 \mathrm{GHz}$ are compared in Fig. 5. A very good agreement among the methods is found, confirming that a good accuracy can be obtained through the Mixed-Methods approach.

The details of the computational performance are summarized in TABLE I.

The Mixed-Methods approach has been extended up to $\mathrm{X}$-band and the relative results are reported in the next section.

\section{RESULTS}

\section{A. L-band Simulation}

As already mentioned the structure was initially simulated at $1.5 \mathrm{GHz}$. The measurements originally made in 1975, after the antenna commissioning, were performed at S-band $(2.115 \mathrm{GHz})$, consequently a direct comparison between the two is not possible. Nevertheless a few considerations are worth to be made.

This antenna exhibits a relatively high Side Lobe Level (SLL). The side lobes are located at $\pm 0.5 \mathrm{deg}$ and have a value between $-12 \mathrm{~dB}$ and $-15 \mathrm{~dB}$. This characteristic can be observed in Fig. 6 which shows some results of the first measurements. The original documentation describes this behavior as a characteristic of this particular design and it was evident since the preliminary measurements of the downscaled model. This peculiar characteristic has been well predicted by our simulations as can be observed in Fig. 7 . 
TABLE I.

COMPUTATIONAL PERformance at $1.5 \mathrm{GHZ}$

\begin{tabular}{|c|c|c|c|c|}
\hline \multicolumn{5}{|c|}{$\begin{array}{c}\text { Method of Moments (MLFMA) } \\
\text { "one-shot" approach }\end{array}$} \\
\hline $\begin{array}{c}\boldsymbol{N}^{\circ} \\
\text { unknowns }\end{array}$ & $\begin{array}{c}\boldsymbol{N}^{\circ} \\
\text { threads }\end{array}$ & $\boldsymbol{R A M}$ & Elapsed Time & $\begin{array}{c}\boldsymbol{N}^{\circ} \\
\text { iterations }\end{array}$ \\
\hline $9,321,135$ & 16 & $170 \mathrm{~GB}$ & $17 \mathrm{~h}$ & $\begin{array}{c}293 \\
\text { (res. 5.0E-03) }\end{array}$ \\
\hline \multicolumn{5}{|c|}{$\begin{array}{c}\text { Iterative Physical Optic } \\
\text { applied to the double reflector system }\end{array}$} \\
\hline $\boldsymbol{N}^{\circ}$ facets & $\begin{array}{c}\boldsymbol{N}^{\circ} \\
\text { threads }\end{array}$ & $\boldsymbol{R} \boldsymbol{A M}$ & Elapsed Time & $\begin{array}{c}\boldsymbol{N}^{\circ} \\
\text { iterations }\end{array}$ \\
\hline 741,185 & 16 & $0.7 \mathrm{~GB}$ & $20 \mathrm{~m}$ & $\begin{array}{c}3 \\
\text { (res. 5.0E-02) }\end{array}$ \\
\hline
\end{tabular}

The maximum level of the cross-polar component is about $37 \mathrm{~dB}$ lower than the co-polar component (Fig. 6). The simulations results (Fig. 7) are once again in a good agreement with the measurements result.

Because of the feeding system design, the antenna geometry is symmetrical (at least theoretically) only with respect to the horizontal plane. For this reason the radiation pattern is expected to be quite symmetrical on the elevation plane, whereas it should show a certain level of asymmetry on the azimuth plane. This characteristic is confirmed by the measurements and can be observed in the simulation results as well (Fig. 8).

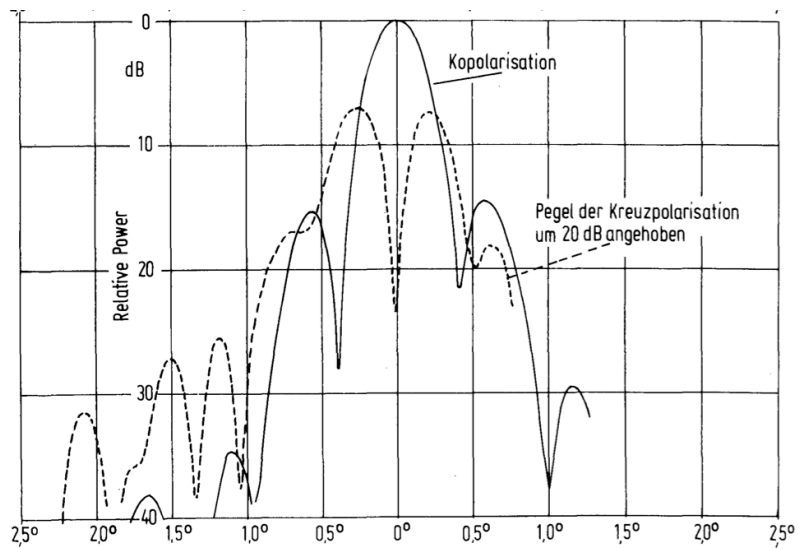

Fig. 6. Measured relative gain for the horizontal polarization in the elevation cut. Frequency $=2.115 \mathrm{GHz}$. The solid line represents the co-polar component, whereas the dashed line represents the crosspolar component. The cross-polar component value is increased by $20 \mathrm{~dB}$ to enhance the readability.

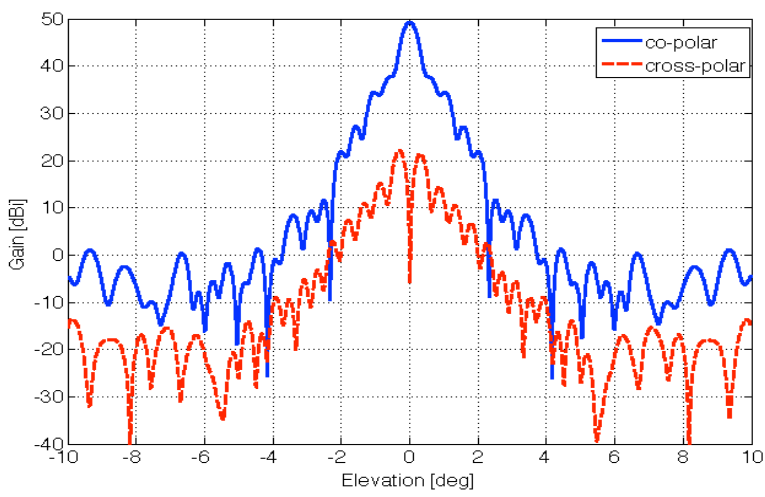

Fig. 7. Simulated radiation pattern for the horizontal polarization on the plane phi $=90 \mathrm{deg}$ (elevation cut). Frequency $=1.5 \mathrm{GHz}$. The solid line represents the co-polar component, whilst the dashed lines is the cross-polar component.

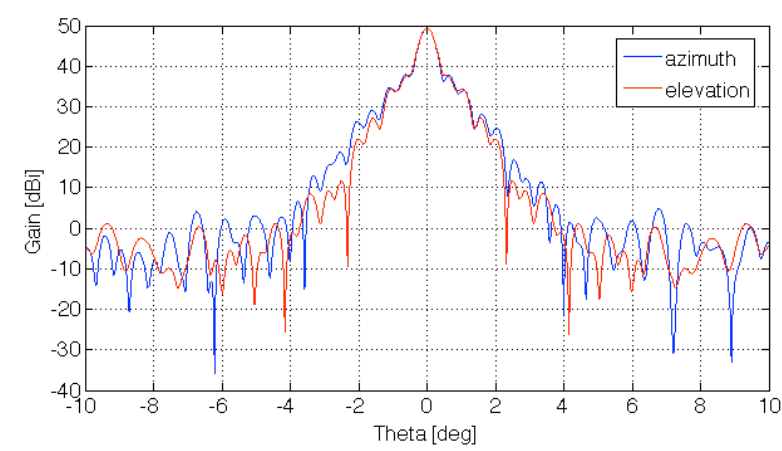

Fig. 8. Simulated radiation pattern for the horizontal polarization Frequency $=1.5 \mathrm{GHz}$. The red line represents the elevation cut, whilst the blue lines represents the azimuth cut.

Finally the maximum gain of the antenna measured in S-band is $51 \mathrm{dBi} \pm 1 \mathrm{~dB}$. The relatively high tolerance is said to be due the measurement setup that generates a high ground reflection and it makes the results scarcely reproducible. Given the latter consideration and the frequency difference, the maximum gain equal to $49.5 \mathrm{dBi}$ obtained by the simulation is an acceptable results that once again confirms the accuracy of the simulation method.

\section{B. X-band Simulation}

The Mixed Method, validated at L-band against the reference full-wave "one-shot" solution, is now used to simulate the antenna at X-band. The huge dimension of the problem - the main reflector diameter is $10^{3} \lambda$ at $10 \mathrm{GHz}$ - sets unprecedented challenges to the IPO solver.

As proved by the result shown in Fig. 5, the whole antenna system can be decoupled in two separate sub-systems:

- Feed and waveguide system;

- Main and Sub reflectors system.

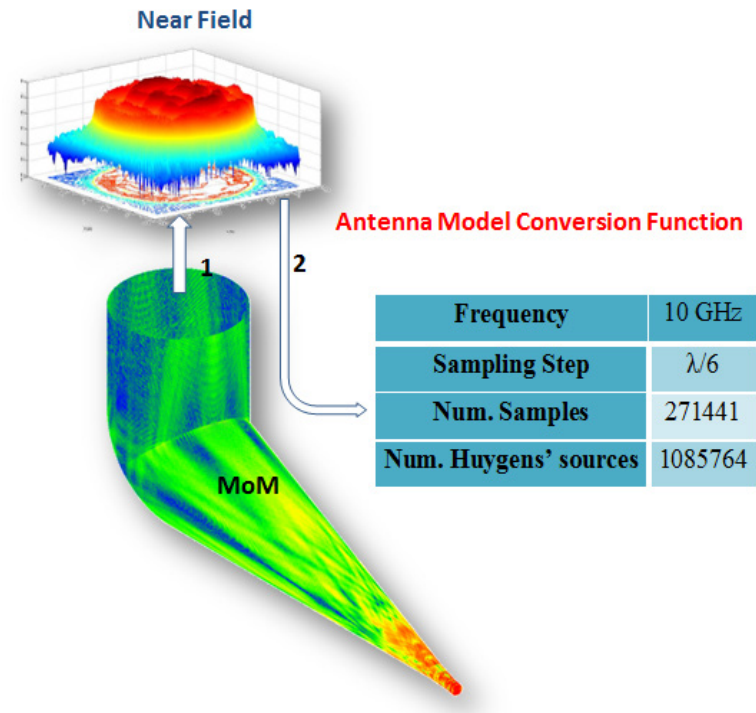

Fig. 9. MoM induced structure currents and the waveguide equivalent currents model at $10 \mathrm{GHz}$. 
Following this assumption, the simulation is carried out through the following computational steps:

1. Full-Wave analysis of the feeding system and calculation of equivalent currents model (Huygens sources can be obtained relying on the equivalence theorem);

2. IPO analysis of the reflectors system by radiating the equivalent current model as the new "impressed" source.

The equivalent current model generation is the intermediate computational process that allows the two procedure's phases to be chained. As shown in Fig. 9:

- A near field map is calculated upon a plane intercepting almost all the power radiated by the launcher $(\lambda / 10$ close to the waveguide aperture);

- Relying on the equivalence theorem, a conversion process is then applied on the near field data in order to get the equivalent current model.

Fig. 10 shows the comparison among the patterns at $10 \mathrm{GHz}$ of the launcher modelled by a full-wave method and the one synthetized as equivalent model in the elevation plane. The good agreement between the curves suggests that the first step of the procedure allows obtaining a very accurate equivalent model of the feeding system.

The IPO total far field radiated by the reflectors system is evaluated as the final step of the simulation procedure. A preliminary result is reported in Fig. 11.

The details of the computational performance at $10 \mathrm{GHz}$ are summarized in TABLE II. The hardware used for the simulation is a server with two Intel ${ }^{\circledR}$ Xeon ${ }^{\circledR}$ CPUs (E5-2690 @ $2.90 \mathrm{GHz}, 16$ cores in total) with $192 \mathrm{~GB}$ of RAM.

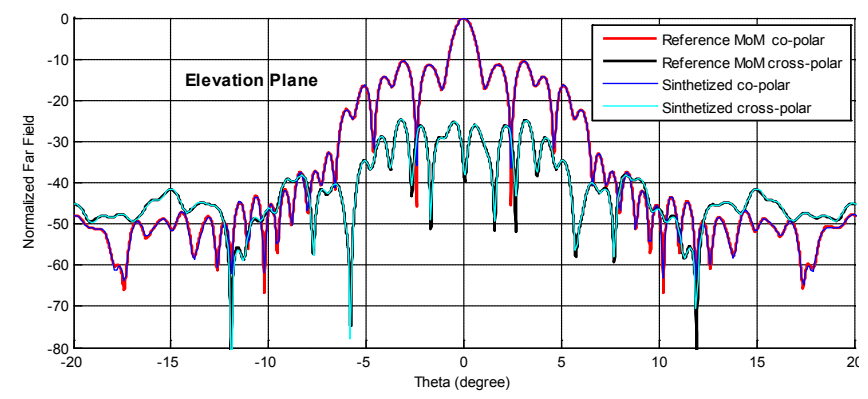

Fig. 10. Equivalent source model vs. reference at $10 \mathrm{GHz}$, elevation plane.

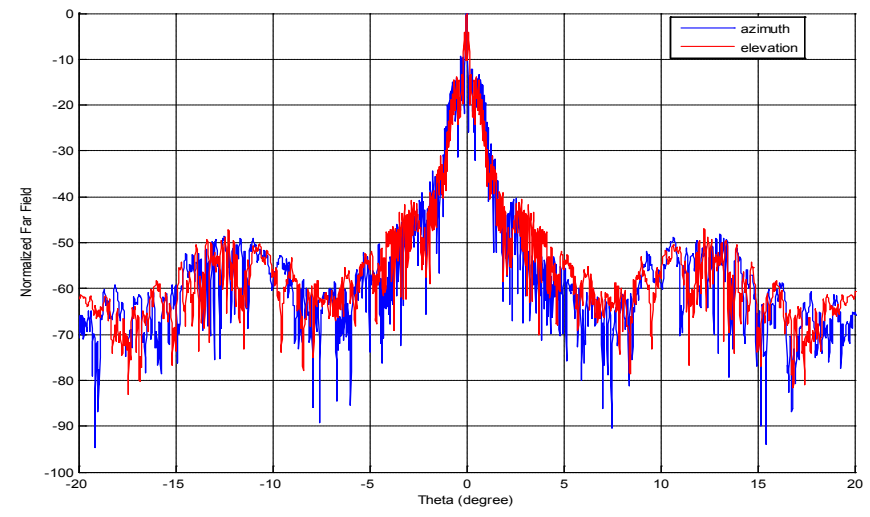

Fig. 11. Simulated radiation patterns (azimuth / elevation planes) at $10 \mathrm{GHz}$.
TABLE II. COMPUtational PERFormance at $10 \mathrm{GHz}$

\begin{tabular}{|c|c|c|c|c|}
\hline \multicolumn{5}{|c|}{$\begin{array}{c}\text { Method of Moments (MLFMA) } \\
\text { applied to the feed and waveguide system }\end{array}$} \\
\hline $\begin{array}{c}\boldsymbol{N}^{\circ} \\
\text { unknowns }\end{array}$ & $\begin{array}{c}\boldsymbol{N}^{\circ} \\
\text { threads }\end{array}$ & $\boldsymbol{R} \boldsymbol{A M}$ & Elapsed Time & $\begin{array}{c}\boldsymbol{N}^{\circ} \\
\text { iterations }\end{array}$ \\
\hline $12,912,796$ & 16 & $189 \mathrm{~GB}$ & $40 \mathrm{~h}$ & $\begin{array}{c}500 \\
\text { (res. 5.0E-02) }\end{array}$ \\
\hline \multicolumn{5}{|c|}{$\begin{array}{c}\text { Iterative Physical Optic } \\
\text { applied to the double reflector system }\end{array}$} \\
\hline $\boldsymbol{N}^{\circ}$ facets & $\begin{array}{c}\boldsymbol{N}^{\circ} \\
\text { threads }\end{array}$ & $\boldsymbol{R A M}$ & Elapsed Time & $\begin{array}{c}\boldsymbol{N}^{\circ} \\
\text { iterations }\end{array}$ \\
\hline $37,743,271$ & 16 & $34 \mathrm{~GB}$ & $66 \mathrm{~h}: 19 \mathrm{~m}$ & $\begin{array}{c}3 \\
\text { (res. 4.6E-02) }\end{array}$ \\
\hline
\end{tabular}

\section{CONCLUSIONS}

The current paper presents the simulation of a $30 \mathrm{~m}$ Near Field Cassegrain antenna. The model has been generated by a real 3D laser acquisition of the reflectors surfaces; consequently the simulation could not take advantage of any kind of symmetry or approximation. The sophisticated MoM acceleration techniques present in the ADF software package, made possible a full-wave simulation of the whole structure at 1.5 GHz. This result was used as reference to validate a new simulation method named Mixed-Methods. By means of this novel approach the antenna has been simulated at $10 \mathrm{GHz}$ and the results are presented.

A question that might arise is why this new method was necessary since existing asymptotic techniques are already adequate to simulate a structure of such dimensions. The answer resides in the feeding system of this particular antenna setup. As the sub-reflector and, in turn, the main reflector illumination has a great impact on the antenna performance, an accurate simulation of the feeding horn reflector is crucial in order to obtain a precise antenna pattern. Although the existing asymptotic techniques can handle the entire geometry, they lack in the accuracy necessary to proper model the launching section of this antenna and consequently their results were never reliable. The usual asymptotic methods always trade accuracy for the ability to simulate large structures. On the other hand, the new method introduced in this paper, in addition to the possibility to simulate large structures, is able to provide high accuracy on selected parts of the geometry where a high level of detail is required.

\section{REFERENCES}

[1] D. C. Hogg and R. A. Semplak, "An experimental study of near-field Cassegrainian antennas", The Bell Syst. Tech. J., vol. 43, pp. $2677-$ 2704, Nov. 1964

[2] M. Sabbadini, G. Guida, and M. Bandinelli, "The Antenna Design Framework - ElectroMagnetic Satellite", Antennas and Propagation Magazine, IEEE, 2009, Volume 51 issue 2.

[3] F. Vipiana, P. Pirinoli, and G. Vecchi, "A multiresolution method of moments for triangular meshes," IEEE Trans. Antennas Propag., vol. 53, pp. 2247-2258, July 2005.

[4] M. Bercigli, F. Vipiana, P. De Vita, A. Mori, A. Freni, G. Vecchi, R. Guidi, M. Bandinelli, "Hybrid MoM Approaches for High Fidelity and Effective Modeling in Large Antenna Farm and Scattering Problems", The 3rd European Conference on Antennas and Propagation (EuCAP 2009), 23-27 March 2009, Berlin, Germany.

[5] F. Obelleiro, J. L. Rodriguez, and R. J. Burkholder, "An iterative physical optics approach for analyzing the electromagnetic scattering by large open-ended cavities," IEEE Trans. Antennas Propag., vol. 43, no. 4, pp. 356-361, Apr. 1995 